\title{
CRYSTALLOGRAPHIC PROPERTIES AND MECHANICAL BEHAVIOR OF TITANIUM HYDRIDE LAYERS GROWN ON TITANIUM IMPLANTS
}

\author{
E. Conforto ${ }^{1}$, D. Caillard ${ }^{2}$, B-O. Aronsson ${ }^{3}$ and P. Descouts ${ }^{3}$ \\ 1IPMC, Swiss Federal Institute of Technology (EPFL), CH-1015, Lausanne, Switzerland, \\ ${ }^{2}$ CEMES / CNRS, 29 rue Jeanne Marvig, F-31055 Toulouse Cedex 4, France
}

${ }^{3}$ GAP Biomedical, Univ. of Geneva, 20 rue de l'Ecole de Médecine, CH-1211 Genève 4, Switzerland

Keywords: SLA treated titanium - bone-anchored dental implants - transmission and scanning electron microscopy - titanium hydride sub-surface layer - epitaxy

\begin{abstract}
Commercially pure titanium for bone-anchored dental implants, submitted to a sand-blasting and acid-etching (SLA) surface treatment, has been mechanically tested and analyzed by SEM and TEM observations. A face-centered cubic titanium hydride layer grows on the polycrystalline titanium substrate with various epitaxial relationships, whose grains show also epitaxial relationships with each other. Indentations, flexion tests and dislocation analyses indicate that this hydride layer can be plastically deformed.
\end{abstract}

* corresponding author. E-mail address: caillard@cemes.fr 


\section{Introduction}

Materials with good potential for applications in life science have been receiving increasing interest from materials scientists in the last years. Specific materials that require new physical properties have been designed for this purpose. However, in spite of their obvious importance, the exact origin of these properties often remains poorly understood. To be used as implant for bone replacement, biomaterials must exhibit high biocompatibility and long life in the human body (more than 20 years) without any corrosion, fracture or delamination. Good mechanical properties are also required, mostly high resistance to fatigue and wear. Indeed, wear debris delivered in the body causing health problems, as well as structural failure due to excessive plastic deformation or delamination, necessitate the removal and replacement of the implant. Two additional essential points are the fixation and the stability of the implant. The former one is not guaranteed by the chemical interactions with the biological tissue, because this kind of interaction is too weak. On the other hand, the second one depends on good mechanical interlocking. For these reasons the interest for the influence of surface roughness on biological interactions has recently increased, especially for boneanchored implants. Because of that, the choice of biomaterial is very important, and among those used until today, titanium and its alloys treated to exhibit rough surfaces are the best.

The SLA ("Sand-blasting and acid etching") surface treatment of Ti implants developed by Institut Straumann AG aims at increasing the surface roughness, to improve osseointegration and to increase the stability of bone-anchored dental implants. This treatment consists in bombarding the surface with a jet of relatively large $(\sim 250 \mu \mathrm{m}) \mathrm{Al}_{2} \mathrm{O}_{3}$ particles followed by an acid $\left(\mathrm{HCl} / \mathrm{H}_{2} \mathrm{SO}_{4}\right.$ hot solution) etching. This treatment causes a two-level roughness: sub-micron porosity $(0.5$ to a few $\mu \mathrm{m}$ in diameter), superimposed on a $20-50 \mu \mathrm{m}$ roughness. The characterization of the resulting surface roughness by SEM has been made and published by Wieland (1999) and Massaro et al. (2002).

It is well known that a $1-3 \mathrm{~nm}$ thick titanium oxide film forms spontaneously at the surface of $\mathrm{Ti}$ 
and its alloys when exposed to air or water. Much attention has been paid by many authors to characterize it precisely and to compare the thickness of these amorphous films formed on different alloys submitted to different surface treatments (see, e. g. Massaro et al., 2002). However, the influence of the treatment chosen for the implant surface can extend beyond the oxide/implant interface. Previous works (Aronsson et al., 2001), on the chemical characterization of the commercially pure $\mathrm{Ti}$ surfaces treated by SLA, determined the presence of hydrogen in the subsurface layer after acid etching. The authors formulated the hypothesis of the formation of another phase, a titanium hydride. If confirmed, this hydride layer could be responsible for the mechanical properties of the Ti surface after SLA treatment, and also contribute to those of the surface/bulk interface.

In the present work, we identified the sub-surface layer and studied its crystallographic relationship with the Ti substrate as well as its internal crystallographic structure. The influence of the latter on the surface layer cohesion as well as on the surface layer/substrate adherence and their mechanical behavior are discussed, with reference to preliminary mechanical tests.

\section{Experimental Procedure}

Commercially pure polycrystalline titanium disks, submitted to the SLA surface treatment (Martin et al. 1995), were provided by Institut Straumann AG (Waldenburg, $\mathrm{CH}$ ). Micro-indentation tests were performed on as-received samples with a SOPELEM micro-indenter, equipped with a Vickers pyramid. Loads were applied in the $20-1200 \mathrm{~g}$ range. Flexion tests were also performed in order to strain the surface layer in tension. Surface transformations due to the SLA treatment in as-received samples, as well as strained surfaces and micro-indentation prints, were observed by scanning electron microscopy (SEM) using a field-emission gun. The instrument was a Philips XL-30 FEG microscope, operated at $10 \mathrm{kV}$.

Crystallographic and dislocation analyses were performed by transmission electron microscopy 
(TEM) on cross-sectional specimens. The instruments were a Philips EM-430, operated at $300 \mathrm{KV}$, and a JEOL 2010, operated at $200 \mathrm{kV}$. Cross-sectional specimens for TEM were cut from the same specimens observed by SEM, and prepared by the classical method: two parts cut perpendicularly to the surface were sandwiched together with an epoxy glue, surface against surface. TEM crosssections were obtained using mechanical polishing and ion milling.

\section{Results}

\subsection{Surface roughness and hardness: SEM analyses}

The surface exhibits the same two-level roughness already observed by Wieland (1999), and by Massaro et al. (2002). According to these authors, the largest one (20-50 $\mu \mathrm{m}$ in diameter) is due to the bombardment with $\mathrm{Al}_{2} \mathrm{O}_{3}$ particles. The finer microstructure with cavities of typically 0.5 to few $\mu \mathrm{m}$ in diameter, which can be seen in figs. 1 and 2 , is due to the acid etching.

Figure 1 shows an indentation print formed under low load $(20 \mathrm{~g})$. It is unsymmetrical because of the roughness of the original surface. From the shape of the indenter, its calculated depth lies in the 2-3 $\mu \mathrm{m}$ range. Several features typical of a heavy plastic deformation are present: regular crushed zones reproducing the pyramid shape, and pad all around it. A few cracks can also be seen, in the pad and in the left side of the image, which have a ductile aspect.

A bending test has been performed, for which the surface layer has been strained by $2 \%$ in tension. The surface has been subsequently observed by SEM. The images reveal a few cracks perpendicular to the straining axis, as in fig. 2. These cracks are $10 \mu \mathrm{m}$ long in average, and homogeneously distributed. A precise measurement of their widths (from $30 \mathrm{~nm}$ to $60 \mathrm{~nm}$ ) and density (one crack every $20 \mu \mathrm{m}$ along the straining axis) shows that they account for less than $20 \%$ of the total strain. Accordingly, the remaining $80 \%$ must be accommodated either by micro-cracks narrower than $5 \mathrm{~nm}$, or by plastic deformation. It is important to note that no delamination is observed in the vicinity of cracks or indentation prints, which indicates a good adherence between the substrate and the hydride 
layer.

\subsection{Cross-sectional TEM on Ti-SLA samples}

\subsubsection{Identification of the sub-surface layer}

Fig. 3a is a low-magnification cross-sectional TEM image showing both sides of the specimen (cross-sectional sample). The presence of tips and valleys confirms the profile described by the SEM observations. The interface with the Ti substrate is found at approximately $2-3 \mu \mathrm{m}$ below the bottom of each valley. The total thickness of this layer is in the 3-6 $\mu \mathrm{m}$ range. It is polycrystalline and contains polygonal and some columnar grains.

Table 1: Identification of the diffraction spots through their Miller indices and interplanar distances, obtained from patterns (Figs. 3b and c) on different tips belonging to the sub-surface layer.

\begin{tabular}{|c|c|c|}
\hline spot \# & hkl & Interplanar distance $(\stackrel{\AA}{\mathbf{A}})$ \\
\hline 1 & 111 & $2.54 \pm 0.03$ \\
\hline 2 & 200 & $2.22 \pm 0.02$ \\
\hline 3 & 220 & $1.57 \pm 0.02$ \\
\hline 4 & 311 & $1.34 \pm 0.01$ \\
\hline 5 & 222 & $1.29 \pm 0.01$ \\
\hline 6 & 331 & $1.01 \pm 0.01$ \\
\hline 7 & 420 & $0.99 \pm 0.01$ \\
\hline
\end{tabular}

In order to characterize the crystal structure of this layer, electron diffraction patterns were collected from different tips (Figs. $3 b$ and 3c). The reflections in these data allow us to identify the complete set of crystallographic planes belonging to a face centered cubic (FCC) structure. The identification of the diffraction spots located along rings, with the corresponding diffracting planes through their Miller indices, as well as their interplanar distances are summarized in Table 1.

These data fit very well with those for the $\mathrm{FCC} \mathrm{TiH}_{1.971}$ phase (JCPDS card 07-0370), belonging to the space group $\operatorname{Fm} 3 m(225)$ with lattice parameter $a=4.44 \AA$. This result is in agreement with 
previous chemical analyses of Aronsson et al.(2001), which indicated a high average hydrogen concentration in the layer. Data may also correspond to several $\mathrm{TiH}_{\mathrm{x}}$ phases $(1.9<\mathrm{x}<2)$ slightly tetragonal below room temperature and FCC above (card 25-0983). $\mathrm{TiH}_{2}$ and $\mathrm{TiH}$ can be excluded owing to their strong tetragonality (respectively $\mathrm{c} / \mathrm{a}=1.34$ and $\mathrm{c} / \mathrm{a}=1.09$, see cards $09-0371$ and 401244), as well as the orthorhombic $\delta$ - $\mathrm{TiH}$ and $\mathrm{TiH}_{0.71}$ phases (cards 44-1217 and 40-0980). In addition, no other known Ti-based compound (carbide, nitride, oxide...) corresponds to the above experimental data.

\subsubsection{First set of crystallographic relationship between hydride layer and Ti substrate}

This section deals with the relative orientations of the unit cells of the hydride with respect to that of the metal. Fig. 4a shows a TEM cross-sectional, bright-field view of a hydride tip ( $\mathrm{n}^{\circ} " 1 "$ in Fig. 3a) and its interface with the Ti substrate. The electron diffraction pattern of the interface (Fig. 4b) simultaneously shows both structures in zone axis: the hexagonal [0110] direction in $\alpha$-Ti and the [011] direction in the FCC hydride, that are parallel within $0.5^{\circ}$. The main diffraction spots are numbered 1 - 4. The same spots can be seen, resolved, in the high resolution diffraction pattern of Fig. 4c. Using them we obtain, separately, dark field images of the two slightly misoriented adjacent grains of the FCC hydride phase (Fig. 4d and 4e), and of the $\alpha-$ Ti substrate (Fig. 4f). Spot \#1 represents, simultaneously, the diffraction of (200) planes in the FCC structure and (0002) planes in the HCP one. Spots \#3 represents the superposition of $(\overline{2} 2 \overline{2})$ and $(1 \overline{1} \overline{2} \overline{2})$ reflections and spot \#4, the superposition of $(02 \overline{2})$ and $(1 \overline{1} 20)$ ones, as seen in fig. 4c. The proximity between the closely separated diffracted spots of the two structures shows that the corresponding dense planes are almost parallel and equidistant. The indexing of the different diffracting planes, deduced from fig. 4, are given in table 2 .

Table 2: Identification of the diffraction spots obtained at the $\mathrm{TiH}_{1.971} / \alpha$-Ti interface. The phase at which they belong as well as their corresponding Miller indices and interplanar distances are indicated.

\begin{tabular}{|l|l|l|l|}
\hline spot \# & phase & hk(i)l & Interplanar distance $(\AA)$ \\
\hline
\end{tabular}




\begin{tabular}{|l|l|c|c|}
\hline & & & (experimental) \\
\hline 1 & FCC-hydride & 200 & $2.22 \pm 0.02$ \\
& $\alpha-\mathrm{Ti}$ & 0002 & $2.32 \pm 0.02$ \\
\hline 2 & FCC-hydride & 111 & $2.54 \pm 0.03$ \\
\hline 3 & FCC-hydride & 220 & $1.56 \pm 0.02$ \\
& $\alpha-\mathrm{Ti}$ & $\overline{2} 110$ & $1.49 \pm 0.01$ \\
\hline 4 & FCC-hydride & 222 & $1.28 \pm 0.01$ \\
& $\alpha-\mathrm{Ti}$ & $\overline{2} 112$ & $1.23 \pm 0.01$ \\
\hline
\end{tabular}

Other crystallographic coincidences can be found while establishing the complete set of crystallographic relationships for both structures simultaneously. They are summarized in table 3 .

Table 3: First set of epitaxial relationships between the Ti substrate and the hydride layer

\begin{tabular}{|c|c|}
\hline epitaxial relationships & remarks \\
\hline$(200) / /(0002)$ & Difference of 4.3\% between interplanar distances \\
\hline$(0 \overline{2} 2) / /(1 \overline{1} 0)$ & Difference of $4.7 \%$ between interplanar distances \\
\hline$(2 \overline{2} \overline{2}) / /(1 \overline{1} 22)$ & Difference of 3.2\% between interplanar distances \\
$(2 \overline{2} 2) / /(11 \overline{2} 2)$ & and misorientation of $3^{\circ}$ \\
\hline$[022] / /[1 \overline{1} 00]$ & common directions, \\
\hline
\end{tabular}

\subsubsection{Columnar grains: second set of crystallographic relationships}

Columnar grains can also be observed in hydride tips e.g. in the bright field TEM image of Fig. 5a (tip $\mathrm{n}^{\circ} 2$ from Fig. 3a). They are practically perpendicular to the interface. From this area, two electron diffraction patterns were obtained, at the same tilt angles. The first one was obtained from the Ti substrate oriented in [0001] zone axis (Fig. 5b). The identification of the diffraction spots and their corresponding diffracting atomic planes are numbered and listed in Table 4. The second is the electron diffraction pattern corresponding to the hydride tip and its interface with the Ti substrate, shown in Fig. 5c. The indexing of those diffraction spots is also listed in Table 4. We observe that the diffraction spots from $\{111\}$ planes in the FCC structure (spots \#6 and 9) coincide spatially with 
those from $\{1100\}$ planes in the $\alpha-\mathrm{Ti}$ (\#10 and \#11, respectively).

A careful analysis of dark field images reveals that columnar hydride grains are in contrast by groups, or families, according to the diffraction spots chosen to produce the image, as shown in fig. 5 d - f. For instance, the same columnar hydride grains (labeled "A") are in dark-field contrast when using spots \#7 (FCC-hydride ( $\overline{3} 1 \overline{1})$ ) and \#9 (FCC-hydride (111)). Family "B" is in contrast when using spots \#2 (FCC-hydride (0 $\overline{2} \overline{2})$ ) and \#4 (FCC-hydride (13 $\overline{3})$ ), and Family "C", when using spots \#3 (FCC-hydride (11 $\overline{3})$ ) and \#6 (FCC-hydride (111)). For two of these families (A and C), the diffracting planes form the same type of zone, with axes respectively [11 2 ] and [2 11$]$. The third one (B) forms a [611] zone axis. In fact, the diffraction pattern in Fig. 5c shows the superposition of these three zone axes, rotated in the plane of the sheet with respect to each other. The reason is probably that the transformation of $\alpha$-Ti to FCC-hydride produces several variants. This means that the three families of hydride grains are in epitaxial relationship with one grain of the Ti substrate. Consequently, they are in epitaxial relationship with each other. Fig. 6 shows this relationship in three dimensions. The three variants have another common direction of $<220>$ type, out of the plane of the former ones, namely [101] (A) // [011] (B) // [101] (C), within $2^{\circ}$. The epitaxy relationships between A - C columnar grains and the Ti substrate are different from those reported in \$3.2.2. They are detailed in table 5.

Table 4: Identification of diffraction spots from the Ti substrate (spots \#10 - 12, Fig. 5b) and from the titanium hydride tip (spots \#1 - 9, Fig. 5c).

\begin{tabular}{|c|c|c|c|}
\hline Spot \# & Phase & hk(i)l & $\begin{array}{c}\text { Family of grains highlighted } \\
\text { in dark field images }\end{array}$ \\
\hline 1 & FCC-hydride & $0 \overline{2} \overline{2}$ & $\mathrm{C}$ \\
\hline 2 & FCC-hydride & $0 \overline{\overline{2}} \overline{2}$ & $\mathrm{~B}$ \\
\hline 3 & FCC-hydride & $1 \overline{\overline{3}}$ & $\mathrm{C}$ \\
\hline 4 & FCC-hydride & $1 \overline{3} \overline{3}$ & $\mathrm{~B}$ \\
\hline 5 & FCC-hydride & $1 \overline{3} \overline{1}$ & $\mathrm{~A}$ \\
& & $1 \overline{3} \overline{1}$ & $\mathrm{C}$ \\
\hline 6 & FCC-hydride & $11 \overline{1}$ & $\mathrm{C}$ \\
\hline
\end{tabular}




\begin{tabular}{|c|c|c|c|}
\hline 7 & FCC-hydride & 311 & A \\
\hline 8 & FCC-hydride & $2 \overline{2} 0$ & A \\
\hline 9 & FCC-hydride & 111 & A \\
\hline 10 & $\alpha-T i$ & $10 \overline{1} 0$ & \\
\hline 11 & $\alpha-\mathrm{Ti}$ & $1 \overline{1} 00$ & \\
\hline 12 & $\alpha-\mathrm{Ti}$ & $11 \overline{2} 0$ & \\
\hline
\end{tabular}

Similar simultaneous crystallographic relationships between adjacent grains of the hydride layer, and between these grains and the Ti substrate have been found in other zones. In fact, we have observed that hydride grains of different shapes, close to the interface with the $\alpha$-Ti show an epitaxial relationship with the latter. At the same time, hydride grains far from the interface, although slightly misoriented respect to those at the interface, have at least one crystallographic direction in common with them. This is illustrated in Fig. 7. A smooth tip ( $n^{\circ} 3$ from Fig. 3a) constituted by polygonal FCC hydride grains is shown in the TEM bright field image of Fig. 7a. The $\alpha$-Ti substrate is oriented in the $[\overline{2} 4 \overline{2} 3]$ zone axis. Polygonal FCC grains close to the interface with the substrate are oriented, for the same tilts, in the [112] zone axis, as we observe in the diffraction pattern of Fig. 7b. This means that [112] // [ $\overline{2} 4 \overline{2} 3]$. Moreover, the neighbor grains closer to the surface of this tip are oriented in the [114] zone axis (Fig. 7c) for the same tilt conditions. Thus, even if they are not in contact with the substrate, they show a crystallographic relationship with their FCC neighbors. The FCC grains shown in dark field images of Figs. $7 b$ and $7 \mathrm{c}$ have a common $<220>$ direction, corresponding to circled spots in the corresponding diffraction patterns. This situation is similar to that previously described in Fig. 6.

Table 5: Second set of epitaxy relationships between the Ti subtrate and the hydride layer

\begin{tabular}{|c|c|}
\hline epitaxy relationships & remarks \\
\hline$(111) / /(1 \overline{100)}$ & Difference of 1\% between interplanar distances \\
\hline$(2 \overline{2} 0) / /(11 \overline{2} 0)$ & Difference of 4.7\% between interplanar distances \\
\hline$(131) / /(01 \overline{1} 10)$ & Difference of 3\% between interplanar distances \\
& and misorientation of $2^{\circ}$ \\
\hline$[11 \overline{2}] / /[0001]$ & Common directions, different interplanar distances between \\
& corresponding planes \\
\hline
\end{tabular}




\subsubsection{Dislocations in the hydride layer and at the hydride/ $\alpha-T i$ interface}

Fig. 8 shows the dislocation structure on both sides of the interface between the $\alpha$-Ti substrate and a large FCC-hydride grain. The density of dislocations is low in the $\alpha$-Ti substrate, but increases towards the interface. They are bowed in the direction of the bulk, which indicates that they have been emitted by the interface, under the internal stress due to the growth of the hydride layer. From observations at various tilt angles, their plane of motion has been identified as a $\{1100\}$ prismatic plane. Contrast analyses showed that their Burgers vector is of the $1 / 3<11 \overline{2} 0\rangle$ type. Their pronounced curvature near the interface allows us to estimate the residual shear stress using the

classical formula $\tau=\frac{\widehat{\mathrm{T}}}{\mathrm{bR}} \approx \frac{1}{2} \frac{\mu \mathrm{b}}{\mathrm{R}}$, where $\tau$ is the local shear stress, $\hat{\mathrm{T}}$ is the dislocation line tension, $\mu$ is the shear modulus, $\mathrm{b}$ is the modulus of the Burgers vector, and $\mathrm{R}$ is the dislocation radius of curvature. This method has been used successfully in previous experiments on Ti crystals of various purities (Farenc et al., 1993). We measure $\mathrm{R}_{1} \sim 100 \mathrm{~nm}, \mathrm{R}_{2} \sim \mathrm{R}_{3} \sim 130 \mathrm{~nm}$. Using $\mu=39.8 \mathrm{GPa}$ and b $=0.295 \mathrm{~nm}$, we obtain $\tau \approx 50 \mathrm{MPa}$. The latter value can be compared with the critical resolved shear stress (CRSS) of prismatic slip in Ti with various impurity contents. According to Okazaki and Conrad (1973) and Naka et al. (1988), this CRSS is in the range 25-100 MPa for commercially pure Ti with a $1500-10000 \mathrm{ppm}$ global impurity concentration $\mathrm{O}+\mathrm{C}+2 \mathrm{~N}$. This value is of the same order of magnitude as the one estimated in our sample. It is accordingly sufficient to induce some plasticity in the Ti substrate near the interface, in agreement with the bowed shape of dislocations discussed above.

In the hydride layer, dislocations form a very dense three-dimensional network typical of a high plastic deformation. Their Burgers vector $\mathbf{b}$ can be determined using the classical out-of-contrast conditions $\mathbf{g} . \mathbf{b}=0$, where $\mathbf{g}$ is the diffraction vector. This analysis is however difficult because the shape and the position of individual dislocations change drastically when the sample is tilted in order 
to reach the required diffracting conditions. The same dislocations can nevertheless be identified in Fig. 9, in the area noted A. The images have been obtained in bright-field conditions, in a grain oriented near the [111] direction. The dislocations are out of contrast with diffraction vectors $\mathbf{g}=$ [200] and $\mathbf{g}=$ [111]. Their Burgers vector is accordingly perpendicular to the plane containing these two directions, namely parallel to the [011] direction. In addition, a double contrast typical of the condition $\mathbf{g . b}=2$ is obtained with $\mathbf{g}=[02 \overline{2}]$. This yields $\mathbf{b}=1 / 2[011]$. Dislocations lie close to the foil plane because their apparent length do not change significantly upon tilting the specimen. Since this plane is close to (111) containing $\mathbf{b}$, they have probably moved by glide. Such a situation is usual in FCC metals and alloys (Friedel, 1964). On the other hand, dislocations noted B to F cannot be analyzed unambiguously. Their contrasts are however consistent with Burgers vectors of the same $1 / 2<110>$ type. It must be noted that dislocations have never been analyzed previously in this material. A dense and isotropic three-dimensional network of $1 / 2<110>$ dislocations gliding in $\{111\}$ planes is typical of a heavily deformed ductile FCC crystal like aluminum.

\section{Discussion}

To study the sub-surface hydride layer, electron diffraction patterns were obtained both over large areas and in specific regions. In all cases, the layer is identified as a $\mathrm{TiH}_{\mathrm{x}}$ compound with $1.9<\mathrm{x}<2$ (most likely value: $\mathrm{x}=1.971$ ) with an FCC structure. The direct contact Ti hydride/ $\alpha$-Ti allows at least two different crystallographic relationships between them, which have been determined. The first relationship has already been reported in two different situations: i) between slightly tetragonal TiH precipitates and $\alpha$-Ti matrix of a titanium alloy containing $1-3 \%$ of hydrogen (Numakura and Koiwa, 1984) and ii) in Ti samples irradiated by hydrogen ions (Zheng et al., 1990). The second relationship has never been reported in the literature, to the knowledge of authors. It can be deduced from the first one by $36^{\circ}$ rotation in the common $(11 \overline{2} 0)$ plane of the HCP-Ti and $(0 \overline{2} 2)$ plane of the FCC-hydride. Both are different from the classical epitaxy between FCC and HCP phases, for which $\{111\} / /\{0001\}$. These quite good epitaxial relationships with misfits varying between $1 \%$ and $4.7 \%$ indicate that the HCP phase transforms fairly easily into the FCC one. However, since the 
transformation of $\alpha$-Ti into FCC-hydride increases the distance between the nearest $\mathrm{Ti}$ atoms by $4.7 \%$, the corresponding expansion must be accommodated by a plastic deformation of the hydride layer. This deformation is probably at the origin of the high density of dislocations found in the hydride phase and in the Ti substrate close to the interface (figs. 8 and 9).

From our observations, we can formulate some hypotheses concerning the shape of hydride grains obtained after phase transformation. First, sand-blasting deforms Ti plastically over some microns in depth, introducing crystalline changes such as polygonization and twins. Second, the acid etching transforms the $\mathrm{Ti}$ in Ti-hydride with a certain memory of the introduced defects: polygonized zones in $\mathrm{Ti}$ result in polygonal zones in $\mathrm{TiH}_{1.971}$ and twins in Ti may result in columnar grains in $\mathrm{TiH}_{1.971}$.

The native oxide layer at the Ti surface (as for silicon and other materials) is, in general, some nmthick and amorphous. When coating a substrate surface, this layer either inhibits the direct contact between the deposited layer and the substrate, or acts as a barrier for their interdiffusion and chemical reaction (see, for instance Conforto and Schmid, 2001). For that reason, native oxide layers are usually removed by an acid and/or plasma etching from the substrate surface. Moreover, ultra vacuum conditions are required to avoid their re-growth during the process,. On the contrary, the SLA treatment simultaneously reduces the native oxide layer and induces the easy epitaxial growth of the hydride layer. Once the etching is interrupted, a new thin native oxide layer is formed at the hydride surface, in contact with air, which stabilizes the former, now sub-surface, hydride. This nanometric amorphous oxide layer cannot however affect the properties of the micrometric crystalline hydride phase. Such a "cheap" but efficient epitaxial growth is the key of the excellent cohesion observed between the two phases, in the vicinity of indentations and cracks. It is a guarantee against any delamination or debris release in the body.

At low loads, the depth of indented regions is of the order of the thickness of the hydride layer. Their aspect shows that the layer has been deformed plastically. This result was unexpected because 
hydrides are usually considered to be highly brittle. It is however in good agreement with a previous study demonstrating the plastic deformation of $\mathrm{TiH}_{\mathrm{x}}(1.58<\mathrm{x}<1.99)$ in uniaxial compression down to room temperature (Irving and Beevers, 1972). Such behavior protects the devices against the effects of accidental scratches. In tension, the layer also exhibits a small amount of plasticity, at least up to about $1 \%$ strain. This rather poor ductility is however largely sufficient if we consider that devices like dental implants are not designed to be highly deformed in normal use conditions.

According to Irving and Beevers (1972), the yield stress in compression should be in the range 150$200 \mathrm{MPa}$ at room temperature. The deformation stress of the hydride layer is probably higher due to its small grain size and large dislocation density. According to the Hall-Petch law (Hall, 1951, Petch, 1953), the strength of a material is increased when its grain or subgrain size is decreased. Note however that in order to benefit from this effect, the tips between adjacent pores must be larger in size than the constituent grains and subgrains. This condition is fortunately satisfied in the present material because the tip size $(1 \mu \mathrm{m})$ is larger than the average width of the columnar or polygonal grains $(\sim 0.2 \mu \mathrm{m}$, as seen in Figs. 5 and 7$)$. Since the tips are, in addition, hardened by a high density of dislocations, they are expected to have a fairly high mechanical strength.

\section{Conclusions}

We have analyzed the crystallographic transformations and the mechanical characteristics of the Ti surface after SLA treatment. We have demonstrated the epitaxial growth of a titanium hydride of composition $\mathrm{TiH}_{\mathrm{x}}$ with $1.9<\mathrm{x}<2$, directly on the titanium substrate. Hydride grains also develop crystallographic relationships between each other. During their growth, hydride grains reproduce the shape of substrate grains. These characteristics guarantee, for this layer, the best possible cohesion and adherence to the $\mathrm{Ti}$ substrate. The plastic deformation of the hydride layer has also been demonstrated, in indentation tests and in tension up to more than $1 \%$ strain. These features constitute the second important physical property of this material for biomedical applications, because the layer can accommodate externally applied stresses without releasing debris in the body. 


\section{Acknowledgements}

Authors thank Institut Straumann AG for providing samples, IMX-EPFL for indentation facilities and CIME-EPFL and CEMES/CNRS-Toulouse for electron microscopy facilities. D.C. thanks EPFL for granting him a leave of absence to pursue part of this research in Lausanne. The financial support from Fonds National Suisse pour la Recherche Scientifique (grant nr. 2134062611.00/1) is gratefully acknowledged.

\section{References}

- Aronsson, B.-O., Hjörvarssojn, B., Frauchiger, L., Taborelli, M., Vallotton, P.-H. and Descouts, P., 2001, J. Biomed. Mater. Res. 54(1), 20.

- Conforto, E. and Schmid, P.E., 2001, Phil. Mag. A 81(1), 61.

- Farenc, S., Caillard, D., and Couret, A., 1993, Acta Metall. Mater. 41, 2701.

- Friedel, J., 1964, Dislocations, Pergamon Press, UK.

- Hall, E.O., 1951, Proc. Phys. Soc. B 64, 742.

- Irving, P.E. and Beevers, C.J., 1972, J. Mater. Sci. 7, 23.

- Martin, J.Y., Schwartz, Z., Hummert, T.W., Schraub, D.M., Simpson, J., Dean, D.D., Cochran D.L. and Boyan, B.D., 1995, J. Biomed. Mater. Res. 29(2), 389.

- Massaro, C., Rotolo, P., De Riccardis, F., Milella, E., Napoli, A., Wieland, M., Textor, M., Spencer, N.D. and Brunette, D.M., 2002, J. Mater. Sci.: Mater. Med. 13, 535.

- Naka, S., Lasalmonie, A., Costa, P., and Kubin, L.P., 1988, Phil. Mag. A 57, 717.

- Numakura, H., and Koiwa, M., 1984, Acta Metall. 32, 1799.

- Okazaki, K., and Conrad, H., 1973, Acta Metall. 21, 1117.

- Petch, N.J., 1953, J. Iron Steel Inst. 174, 25.

- Wieland, M., 1999, “Experimental determination and quantitative evaluation of the surface composition and topography of medical implant surfaces and their influence on osteoblastic cellsurface interactions”, Ph.D. thesis no. 13247, ETH Zürich, Switzerland. 
- Zheng, P., Ruault, M.O., Fournier, D., and Saint-Jacques, R.G., 1990, Journal of Materials Science Letters 9, 75.

\section{Figure Captions}

Figure 1: SEM image of a low-load indentation print. Note the pad around the print (arrows), and the few cracks in the pad and in the left side of the print.

Figure 2: SEM image of the surface layer strained by $2 \%$ in tension after bending. Only a few cracks (arrowed) can be seen along the direction perpendicular to the straining direction (noted SD).

Figure 3: a) TEM cross-sectional bright field image of the Ti surface treated by SLA at low magnification. b) and c): Electron diffraction patterns obtained from two neighboring tips, evidencing an FCC structure. The spot numbers refer to Table 1.

Figure 4: a) TEM cross-sectional bright field image of a tip at its interface with the Ti substrate. b) Electron diffraction pattern of the $\mathrm{TiH}_{1.971} / \alpha$-Ti interface, showing diffraction spots from both structures. c) High resolution diffraction patterns. d) and e): Dark field images of two slightly misoriented $\mathrm{TiH}_{1.971}$ grains using the diffraction from (200) planes. f) Dark field image of the Ti substrate in the same tilt conditions using the diffraction of the (0002) planes. Spot numbers refer to Table 2.

Figure 5: TEM analyses of columnar-shaped grains. a) Bright field image showing grains forming a tip and their interface with the $\alpha$-Ti; b) Electron diffraction patterns of the $\alpha$-Ti substrate; c) Electron diffraction patterns showing the zone axes corresponding to each family of columnar grains; $d$ - f) 
Dark-field images of the families A, B and C of columnar-shaped grains.

Figure 6: Stereographic projection, showing the epitaxial relationships between the three columnarshaped grains and the Ti substrate, in Figure 5. Note that the three variants have a $\langle 220\rangle$ direction in common.

Figure 7: Hydride tip containing polygonal grains: a) Bright field image of the tip with an insert corresponding to the electron diffraction pattern of the Ti substrate. Dark field image of the hydride grains: b) at the interface with $\alpha-\mathrm{Ti}$, in the [112] zone axis (see insert), obtained using the diffraction of $\{111\}$ planes (intense spots); c) close to the extremity of the tip, in the [114] zone axis, using the diffraction of the FCC $\{220\}$ planes. Note that both hydride grains have a common $<220>$ direction (circled spots).

Figure 8: Dislocation structure on both sides of the interface between the $\alpha$-Ti substrate and a large hydride grain. a) Dense network of dislocations on the hydride side. b) Curved dislocations in the $\alpha$ Ti side, which allow one to determine the local stress

Figure 9: Determination of the Burgers vector of dislocations in the hydride layer. Bright-field images near [011] and [110] zone axes (a-e and f-h respectively), showing that dislocations in the region A are out of contrast with diffraction vectors $\mathbf{g}=[200]$ (fig. b), and $\mathbf{g}=$ [111] (fig. g); and in double contrast typical of the condition $\mathbf{g} . \mathbf{b}=2$, with $\mathbf{g}=[02 \overline{2}]$ (fig. $\mathbf{d}$, double contrast is arrowed). 


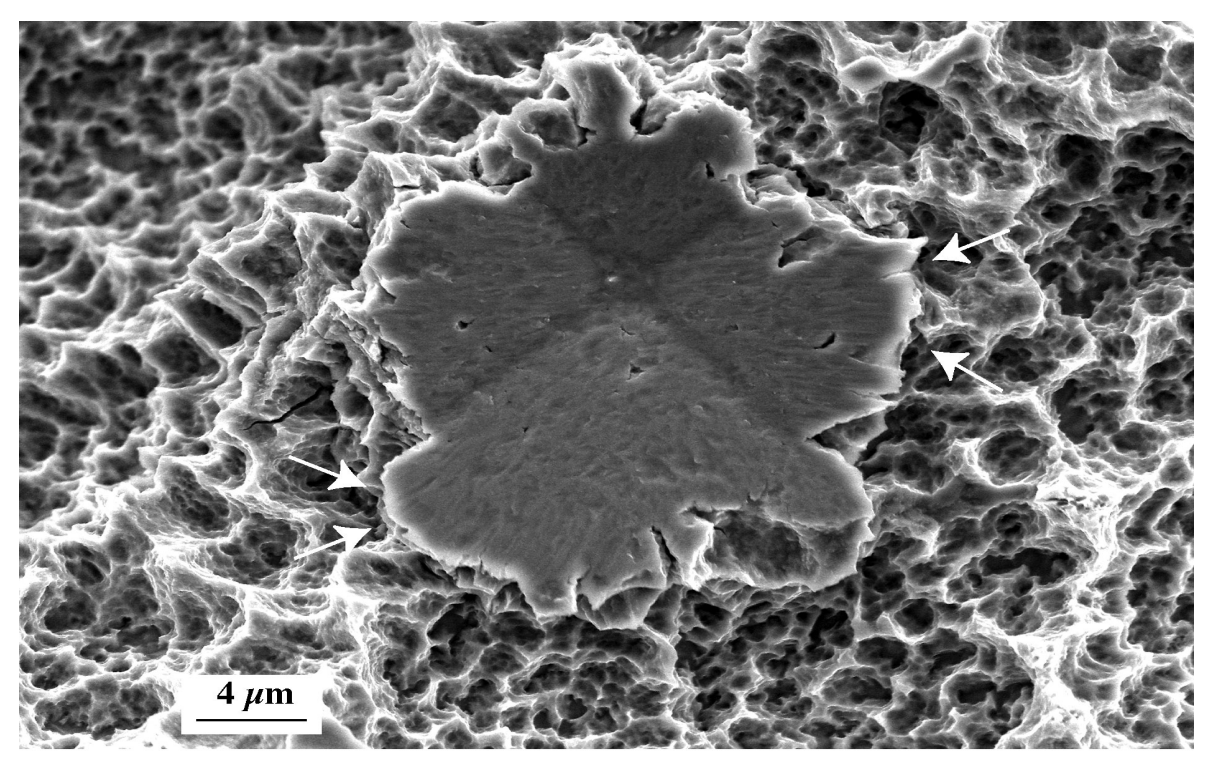

Fig. 1 


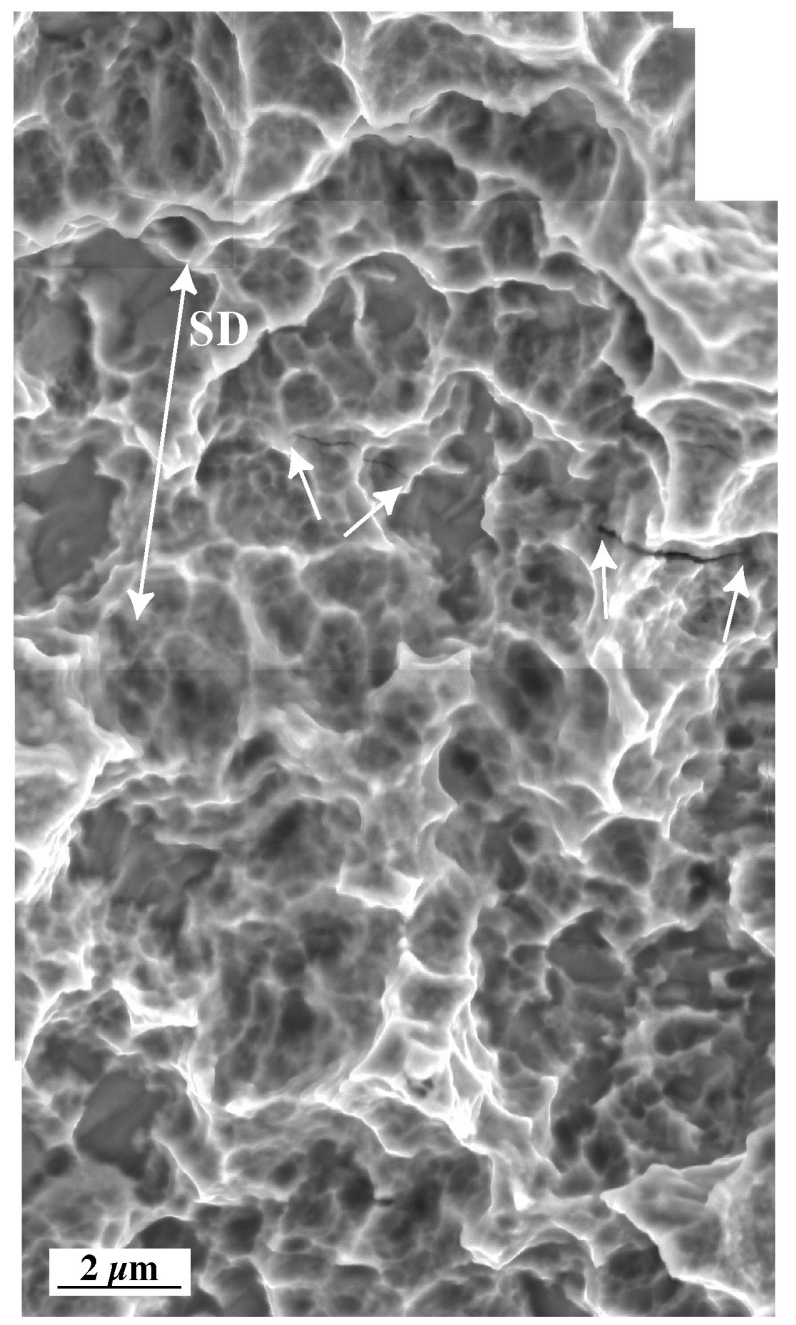

Fig. 2 


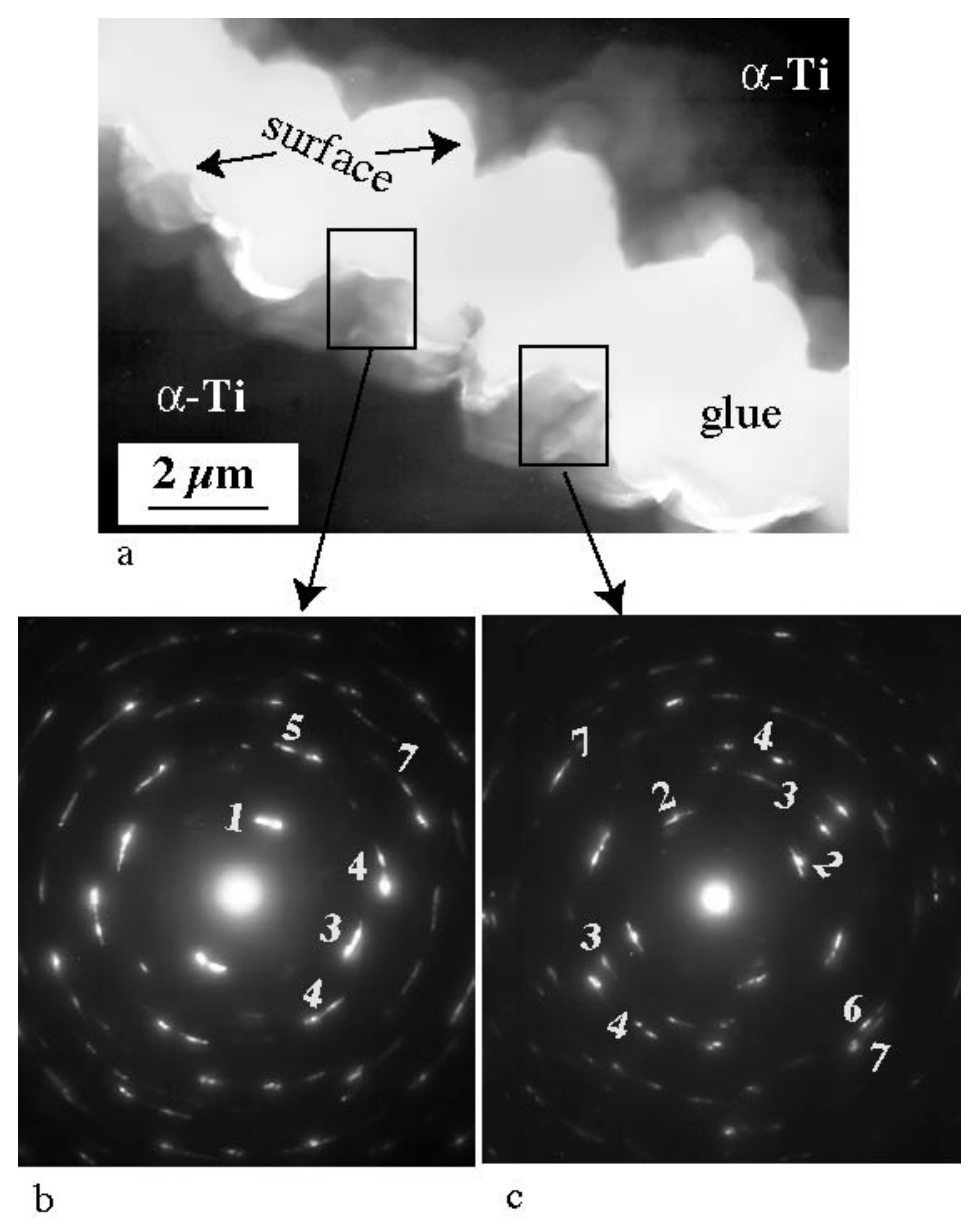

Fig. 3 

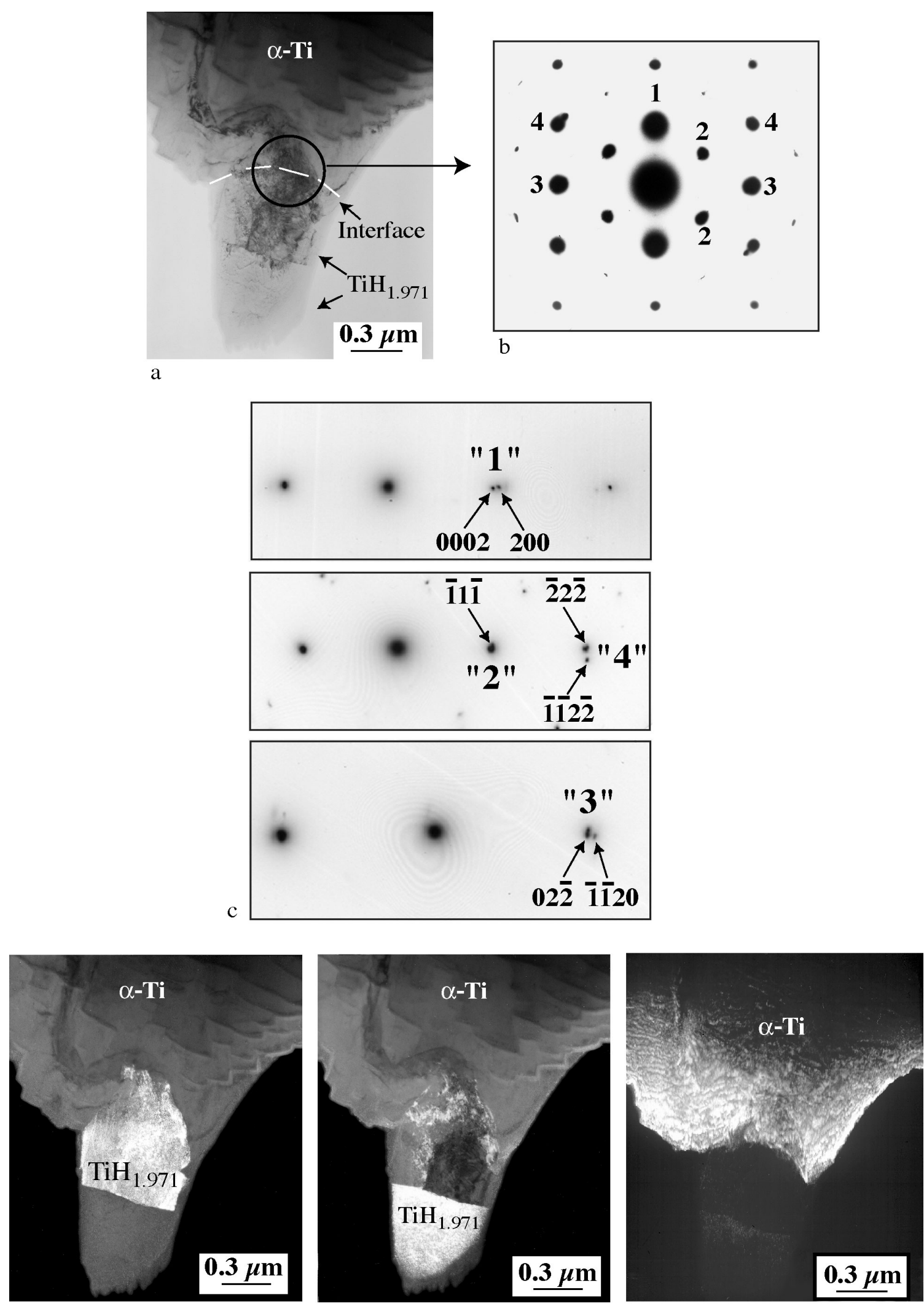

d

e

f

Fig. 4 


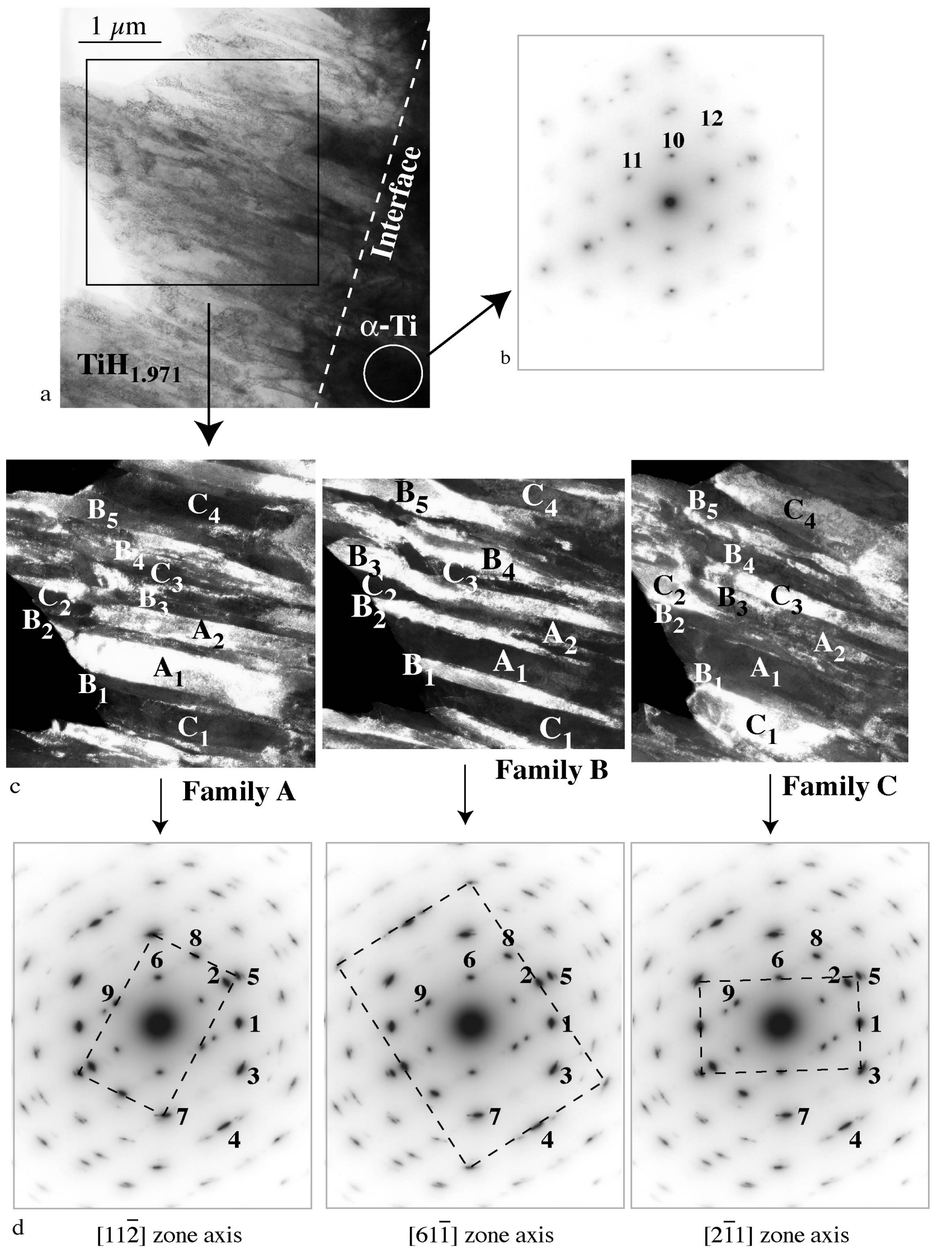

Fig. 5 


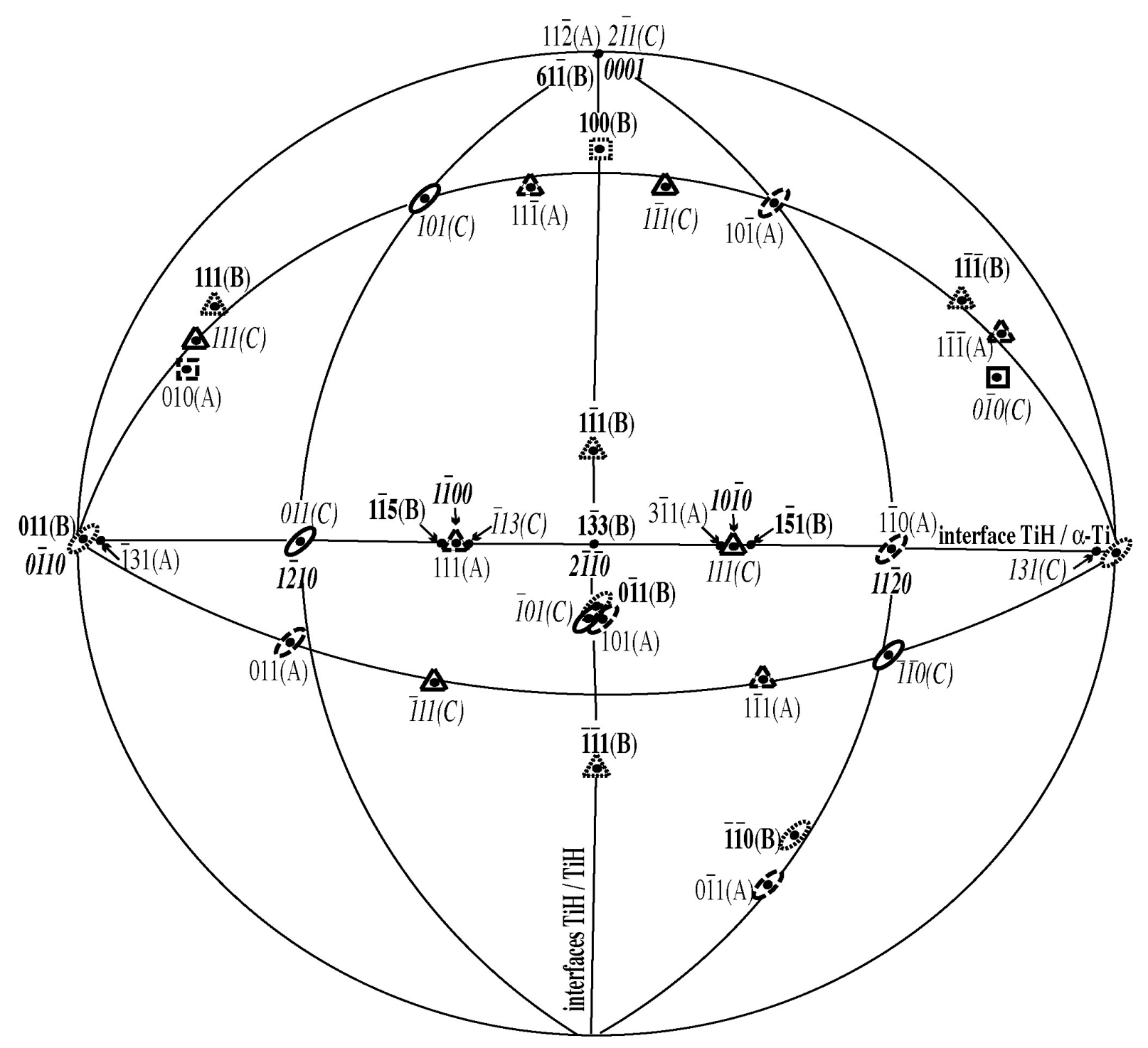

Fig. 6 


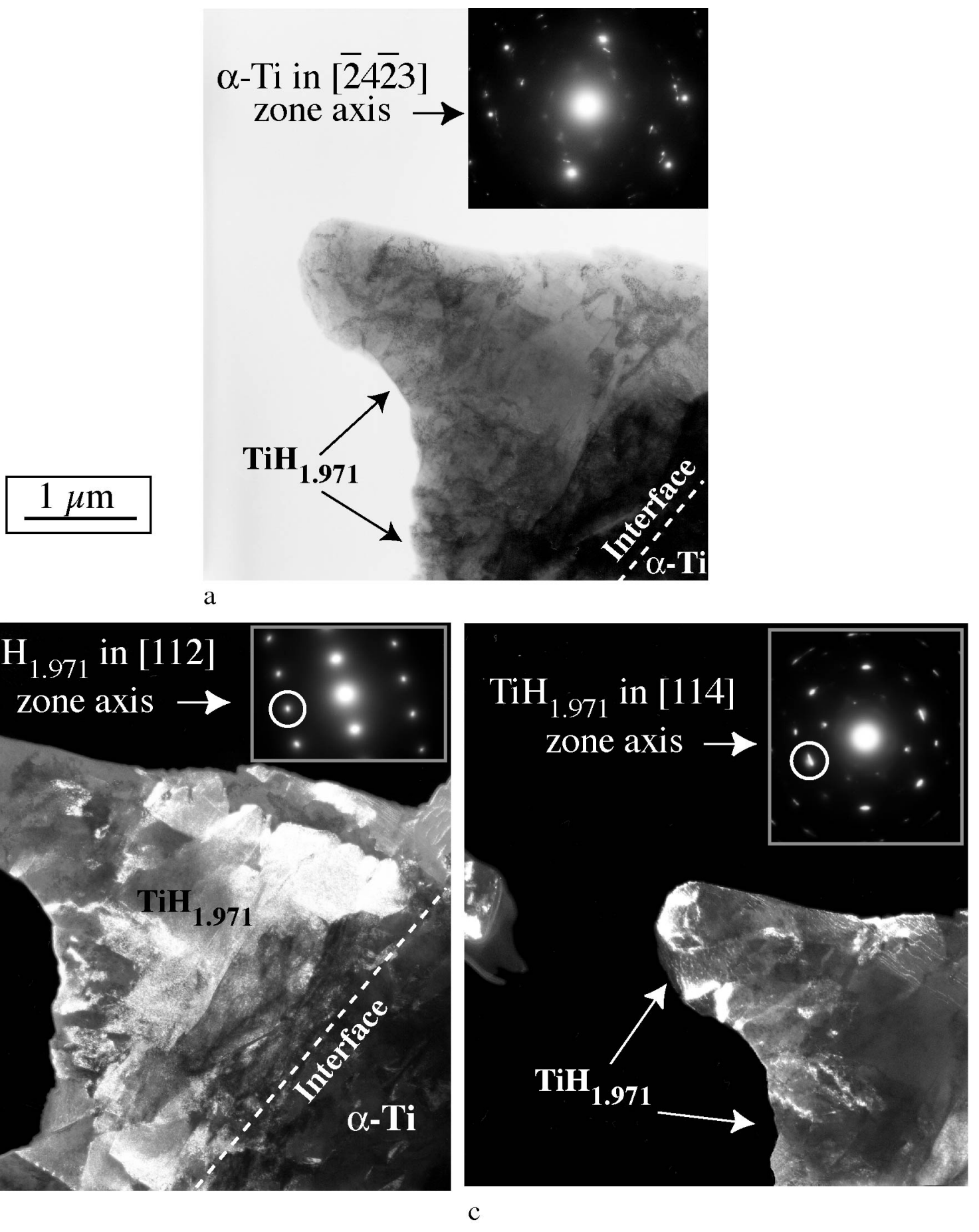

Fig. 7 


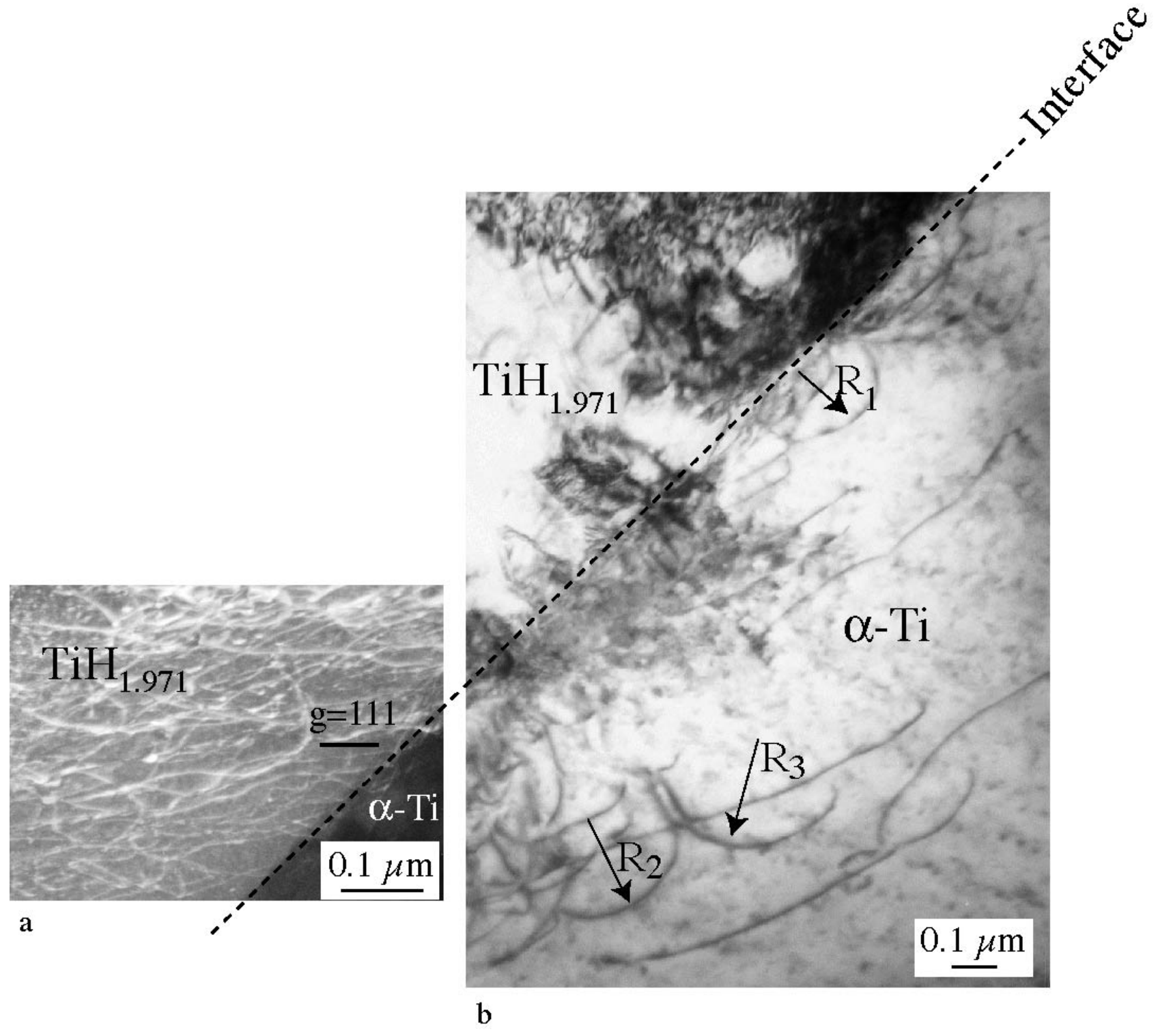

Fig. 8 


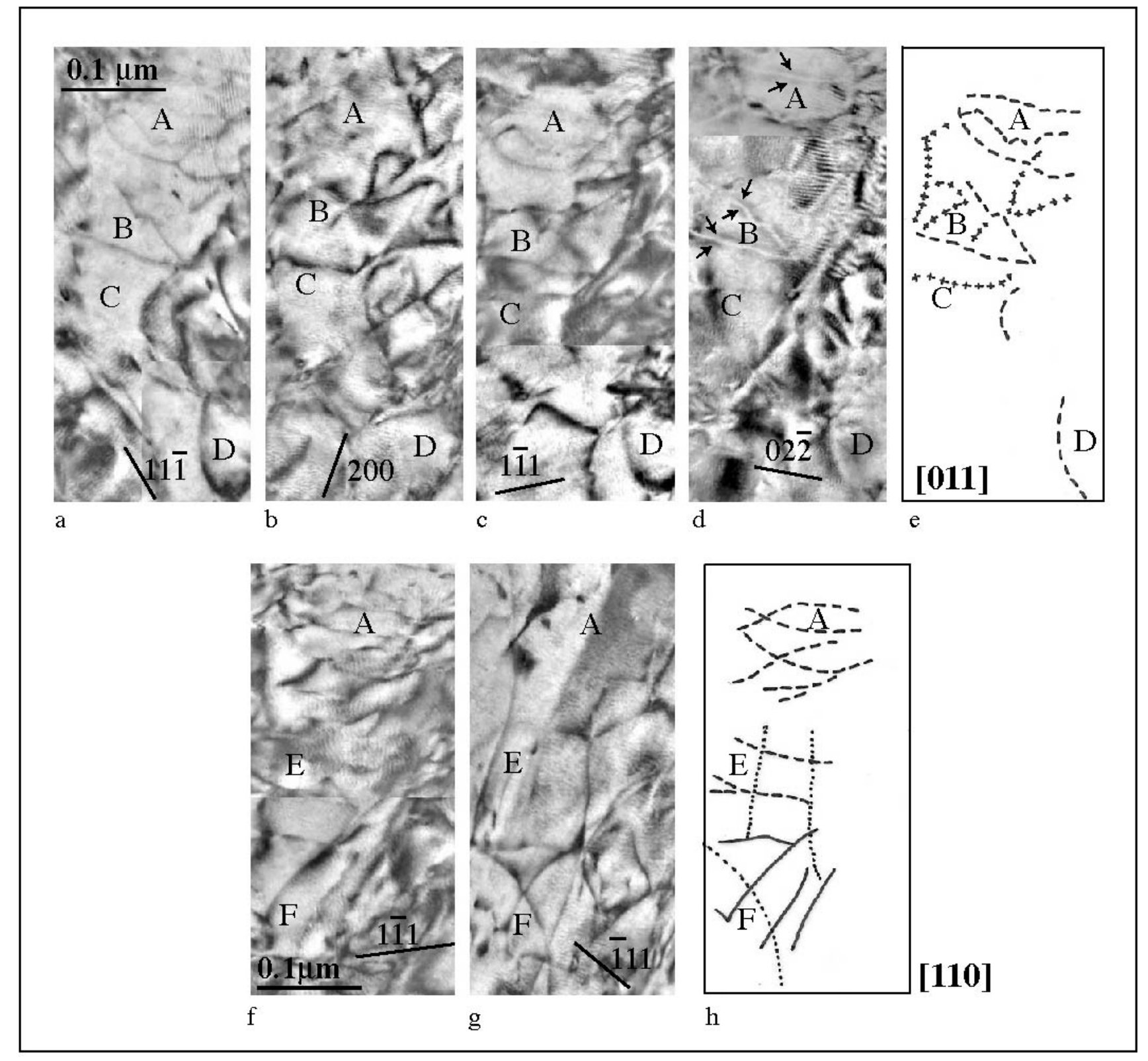

Fig. 9 